\title{
Weathering the Crisis: A Case of Thyroid Crisis with Propranolol-Induced Circulatory Collapse Successfully Treated with Therapeutic Plasma Exchange
}

\author{
Jean Mun Cheah, ${ }^{1}$ Danish Ng, ${ }^{1}$ Ming Yoong Low, ${ }^{2}$ Siew Hui Foo ${ }^{1}$ \\ ${ }^{1}$ Endocrine Unit, Department of Medicine, Selayang Hospital, Ministry of Health, Malaysia \\ ${ }^{2}$ Department of Nephrology, Selayang Hospital, Ministry of Health, Malaysia
}

\begin{abstract}
Thyroid crisis is a life-threatening form of thyrotoxicosis characterized by multi-system dysfunction. Therapeutic plasma exchange has been reported to be effective in removing excessive circulating thyroid hormones. We present a 46-year-old female with recently diagnosed Graves' disease associated with thyrotoxic cardiomyopathy admitted for thyroid crisis complicated by propranolol-induced circulatory collapse, acute kidney injury and ischemic hepatitis. The tachyarrhythmia was refractory to conventional therapy. Initiation of TPE resulted in rapid clinical and biochemical stabilization.
\end{abstract}

Key words: thyroid crisis, circulatory collapse, cardiomyopathy, propranolol, therapeutic plasma exchange

\section{INTRODUCTION}

Thyroid crisis is a potentially lethal complication of thyrotoxicosis if inadequately treated. It is a state of metabolic crisis characterized by multisystem dysfunction due to excess thyroid hormone release. Patients with thyroid crisis should be managed in a multi-modality approach with anti-thyroid drugs (ATDs), inorganic iodide, glucocorticoids and anti-adrenergic drugs. ${ }^{1}$ Propranolol, a non-cardio-selective $\beta$-blocker (NCBB) is an anti-adrenergic agent commonly used to control the sympathomimetic symptoms in thyroid crisis patients due to its additional effect of blocking the peripheral conversion of inactive thyroxine (T4) to the active thyroid hormone, tri-iodothyronine (T3) ${ }^{2}$. However, patients with thyroid crisis may have clinical or subclinical thyrotoxic cardiomyopathy that predisposes them to an exaggerated response to $\beta$-blocker therapy manifesting as circulatory collapse. Therapeutic plasma exchange (TPE) is an alternative treatment for thyroid crisis when life-threatening symptoms are present or conventional medical therapy has failed or is contraindicated. ${ }^{2}$ We report a 46-year-old female with recently diagnosed Graves' disease associated with thyrotoxic cardiomyopathy who presented with thyroid crisis and developed circulatory collapse after administration of $\beta$-blocker and was subsequently treated with TPE.

\section{CASE}

A 46-year-old female with background history of hypertension and type 2 diabetes with recently diagnosed Graves' disease associated with thyrotoxic cardiomyopathy was admitted for thyroid crisis. She was diagnosed with Graves' disease four months ago. She had presented with heart failure along with a seven-month history of palpitations, diaphoresis, weight loss, and tremors. Her free thyroxine (FT4) level was $68.1 \mathrm{pmol} / \mathrm{L}$ (reference range 11.8-23.2) while thyroid stimulating hormone (TSH) level was suppressed. Her anti-thyroid peroxidase (anti-TPO) and anti-thyroglobulin (anti-TG) were positive while thyroid stimulating hormone receptor antibodies (TRAb) was not sent as it was not available in our centre. She was treated with carbimazole, propranolol and furosemide. Unfortunately, the patient defaulted her medications and follow-up. She presented again with a two-month history of progressive exertional dyspnea, orthopnea and bilateral leg swelling. On examination, she was restless, clinically thyrotoxic and tachypneic with a respiratory rate of 22 cycles per minute, her blood pressure (BP) was $155 / 117 \mathrm{mmHg}$, temperature was $37.1^{\circ} \mathrm{C}$. Cardiovascular examination revealed a raised jugular venous pressure, bibasal crepitations in the lungs and bipedal edema up to mid-shin. Electrocardiogram showed atrial fibrillation with rapid ventricular rate of 170 beats per minute. Capillary blood glucose was 5.5 $\mathrm{mmol} / \mathrm{L}$. Chest radiograph showed cardiomegaly with pulmonary congestion (Figure 1). Her free T4 level was $105.3 \mathrm{pmol} / \mathrm{L}$ and $\mathrm{TSH}$ was $<0.01 \mathrm{mU} / \mathrm{L}$ (reference range 0.35- $5.5 \mathrm{mU} / \mathrm{L}$ ). A diagnosis of thyroid crisis precipitated by non-compliance to medication was made with a Burch-Wartofsky score of 60. A loading dose of oral propylthiouracil $600 \mathrm{mg}$, intravenous (IV) hydrocortisone $200 \mathrm{mg}$ and oral propranolol $40 \mathrm{mg}$ were administered. Twenty minutes after the administration of propranolol, the patient developed sudden onset of severe respiratory 
distress, hypotension with a BP of $85 / 40 \mathrm{mmHg}$ leading to emergency intubation. Post-intubation, she progressed into pulseless electrical activity with return of spontaneous circulation after 18 minutes of cardiopulmonary resuscitation. The diagnosis of propranolol-induced circulatory collapse was made and she was admitted to intensive care unit requiring triple inotropic support with IV adrenaline, noradrenaline and dopamine infusions.

A repeat electrocardiogram showed persistent atrial fibrillation with no ST segment changes. Echocardiogram revealed poor heart contractility, global hypokinesia with estimated left ventricular ejection fraction of $30 \%$. She remained in rapid atrial fibrillation with cardiogenic shock despite synchronized cardioversion, digoxin and amiodarone. IV esmolol infusion for control of the tachyarrhythmia had to be discontinued after 3 hours due to worsening hypotension. Her clinical condition continued to deteriorate despite high dose of oral carbimazole $30 \mathrm{mg}$ six hourly, IV hydrocortisone $100 \mathrm{mg}$ eight hourly and Lugol's iodine 10 drops six hourly. She developed ischemic hepatitis, coagulopathy, and anuric acute kidney injury with metabolic acidosis requiring continuous veno-venous hemodialysis (CVVHD). The first cycle of TPE was initiated on the fourth day of hospitalization with two liters of fresh frozen plasma and one liter of human albumin. The patient's hemodynamic status improved significantly within 24 hours allowing tapering of the inotropic support with heart rate reduced to $120-130$ beats per minute. Another two cycles of TPE were performed on day five and day seven of hospitalization followed by resolution of pulmonary congestion and successful extubation by day nine of hospitalization (Figure 2). Inotropes were also tapered off by day eight of hospitalization followed by restoration of sinus rhythm at day 15 . There was no transfusion reaction or hemodynamic instability throughout the three cycles of TPE. Her free T4 level decreased markedly into the normal range (13.8 pmol/L) after three sessions of TPE and continued to fall to $7.3 \mathrm{pmol} / \mathrm{L}$ at day 13 of hospitalization

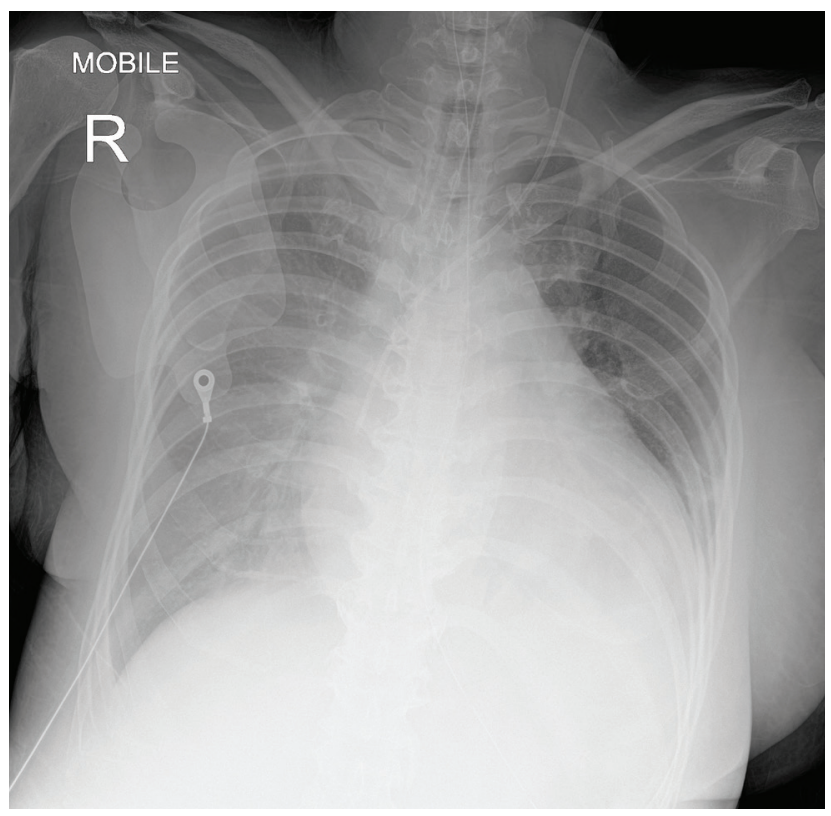

Figure 1. Chest radiograph showing cardiomegaly and pulmonary congestion upon admission. necessitating reduction of carbimazole to maintenance dose of $10 \mathrm{mg}$ daily (Figure 3). Liver and renal dysfunction also resolved by day 16 of hospitalization. The patient's recovery was complicated by critical illness neuropathy post extubation. She was transferred to another rehabilitative facility one month after admission and continued to recover neurologically with intensive physiotherapy. The patient was planned for radioactive iodine therapy in six months as definitive treatment of the Graves' disease.

\section{DISCUSSION}

Thyroid crisis carries a mortality rate of up to $30 \%$ if not recognized and treated promptly. ${ }^{1,2}$ It is characterized by multisystem dysfunction involving mainly cardiovascular, neurological, gastro-intestinal and hepato-biliary systems due to excessive release of thyroid hormone. Hyperthyroidism creates a state of high cardiac output by increasing cardiac contractility, heart rate and decreasing peripheral resistance. It also induces a hyperadrenergic state by amplifying formation and reducing degradation of $\beta$-adrenergic receptors. ${ }^{3}$ Both mechanisms play a compensatory role in maintaining cardiac output of patients with clinical or subclinical thyroid cardiomyopathy during states of stress. Propranolol is an NCBB commonly used in the treatment of thyroid crisis. The administration of propranolol to patients with preexisting clinical or subclinical thyrotoxic cardiomyopathy may result in an uncommon but serious adverse outcome. Administration of NCBB may impede the thyrotoxicosis induced hyperadrenergic state, halting the compensatory mechanism causing significant fall in cardiac output in the setting of stress such as thyroid crisis, leading to circulatory collapse. $^{2}$ Our patient had evidence of pre-existing thyrotoxic cardiomyopathy when she presented with heart failure four months prior to current hospitalization. She developed a drastic drop in BP followed by cardiac arrest shortly after propranolol administration. The temporal association between propranolol administration

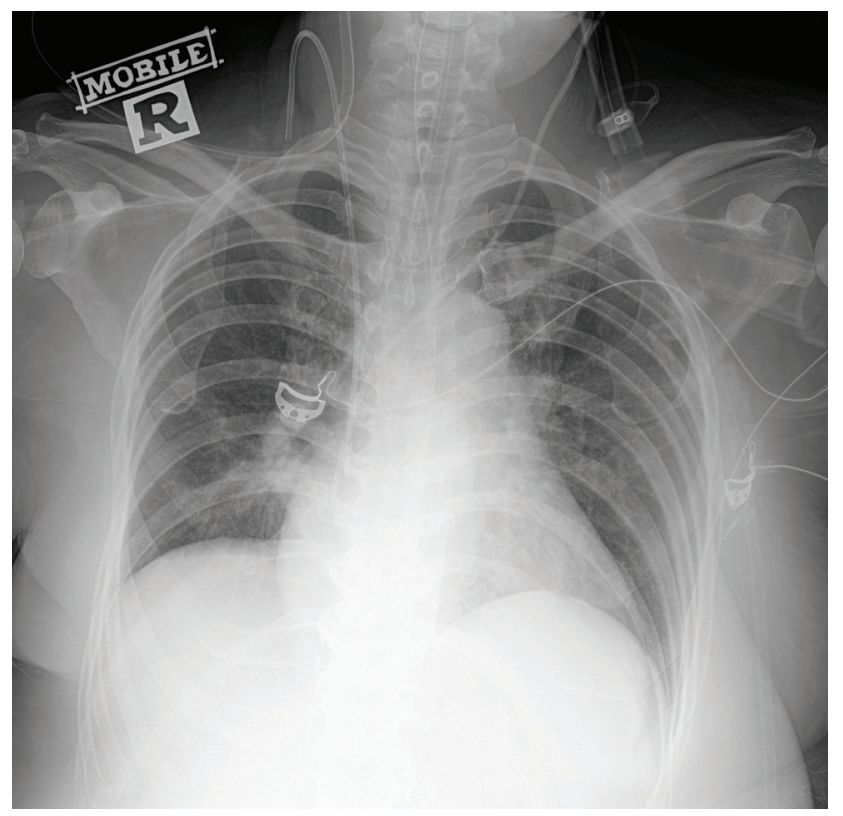

Figure 2. Chest radiograph showing resolution of cardiomegaly with marked improvement of pulmonary congestion by day nine of hospitalization. 


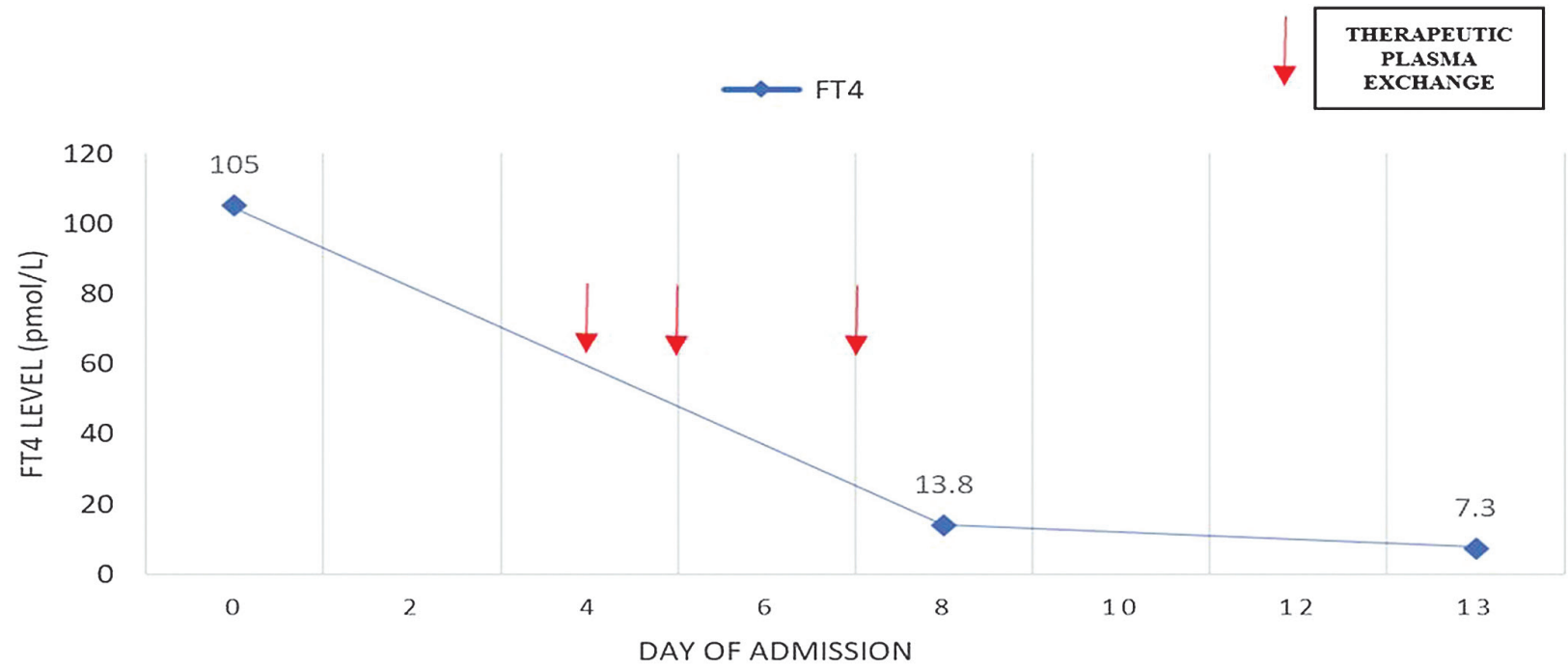

Figure 3. Free thyroxine (FT4) serum concentrations during hospitalization before and after therapeutic plasma exchange (TPE).

and the sudden onset of hemodynamic instability led to the diagnosis of propranolol-induced cardiogenic shock confirmed by the echocardiographic findings of reduced ejection fraction with poor heart contractility. A total of 11 cases of $\beta$-blocker induced circulatory collapse in thyroid crisis have been described in the literature. ${ }^{3}$ Almost all the patients had evidence of pre-existing thyrotoxic cardiomyopathy with five of them having documented low ejection fraction prior to the adverse event. Six of them had cardiac arrest while the rest had hypotension with three fatalities reported. Propranolol was the most commonly used $\beta$-blocker. The presence of thyrotoxic cardiomyopathy especially with low output heart failure may predispose one to an exaggerated response to $\beta$-blocker therapy manifesting as circulatory collapse secondary to cardiogenic shock. However complete cessation of $\beta$-blocker therapy may not be possible due to uncontrolled tachycardia. IV esmolol infusion was used in our patient to control the refractory tachyarrhythmia but it was not well tolerated due to hypotension most probably related to the concomitant CVVHD for anuric acute kidney injury and metabolic acidosis. In a Japanese nationwide survey that compared the use of $\beta_{1}$-selective and nonselective $\beta$-blocker in thyroid crisis, it was found that all deaths among patients with congestive heart failure Killip class three and above were treated with NCCB while those who survived were treated with $\beta_{1}$-selective blocker. ${ }^{4}$ Both esmolol and landiolol are ultra-short acting IV $\beta$-blockers with high cardio-selectivity and short half-life of four to nine minutes as opposed to 23 hours for propranolol. ${ }^{4}$ Due to the ultra-short half-life which allows frequent dose titration and rapid weaning from the $\beta$ blockade effect upon discontinuation, they may be considered as safer alternatives in patients with underlying thyrotoxic cardiomyopathy compared to propranolol especially during thyroid crisis. ${ }^{3,5}$ The Japanese Thyroid Association recommended the use of $\beta_{1}$-selective blocker such as esmolol, landiolol or bisoprolol over NCCB in the treatment of tachycardia in thyroid crisis. IV esmolol or landiolol is preferred over oral bisoprolol if heart rate is more than 150 beats per minute. It can be switched to oral bisoprolol when heart rate is less than 150 beats per minute in Killip class three or below. ${ }^{4}$
The use of TPE in thyroid crisis has been described in the literature as early as 1970s. ${ }^{6}$ The benefits of TPE mainly results from plasma removal of thyroid hormones and their bound proteins, putative autoantibodies, cytokines, catecholamines etc. Although its efficacy is yet to be verified in prospective randomized control trial as thyroid crisis is a rare endocrine emergency, many successful cases of TPE use in thyroid crisis have been reported. ${ }^{6}$ TPE should be considered early in the presence of life-threatening symptoms in thyroid crisis when rapid removal of excess thyroid hormones and its binding proteins is essential as it is the fastest way to produce clinical and biochemical improvement. In our patient, the decision to initiate TPE was made at day two of hospitalization in view of the refractory tachyarrhythmia in shock with onset of renal and liver dysfunction indicating high risk of mortality if urgent measure to reduce the thyroid hormone level was not performed. However, the risk of hemodynamic compromise and the technical difficulties involved with the ongoing CVVHD only allowed the initiation of TPE at day four of hospitalization. Significant clinical improvement was observed within 24 hours after the first cycle of TPE. The FT4 level dropped by $85 \%$ into the normal range after three cycles of TPE over four days. Thyroid function test was not repeated until the end of third cycle of TPE. Clinically, patient continued to improve with each cycle of TPE. The cardiac, liver and renal dysfunction resolved completely 12 days after the initiation of TPE. It was believed that the clinical improvement was predominantly contributed by the TPE as the patient failed to improve within the first 72 hours of presentation when she was on high dose of ATD. Among the reported cases of thyroid crisis successfully treated with TPE, clinical improvement was typically observed within 24 to 72 hours with one to five cycles of TPE. ${ }^{6}$ However, its effect was transitory in nature and conventional treatment with ATDs, glucocorticoids, $\beta$-blocker and inorganic iodide should be administered concomitantly unless contra-indicated to prevent early relapse. Early biochemical improvement in thyroid hormone levels was also frequently observed but its improvement varied between 15 to $78 \%$ depending on baseline thyroid hormone levels. Clinical-biological dissociation is not 
uncommon in which clinical improvement often preceded the decrement in thyroid hormone levels. The overall incidence of adverse events associated with TPE such as hemodynamic instability, transfusion reaction, infectious complications etc., is about $5 \%$. Death was rare and was usually due to the underlying disease. ${ }^{6}$ The Japan Thyroid Association recommended the use of TPE when there is no clinical improvement with conventional therapy within 24 to 48 hours. ${ }^{4}$ The American Society of Apheresis graded the use of TPE in thyroid crisis as category III in which an optimal role is not established and decision should be individualized. ${ }^{7}$ It is also recommended to perform every 24 to 72 hours with $40-50 \mathrm{ml} / \mathrm{kg}$ of replacement fluids until clinical improvement. ${ }^{7.8}$ Both societies recommended the preferential use of fresh frozen plasma which contains T4-binding globulins to albumin as replacement solution. ${ }^{4,7}$ The overall incidence of adverse events associated with TPE is about $5 \% .^{6}$ Notable side effects of TPE include hemodynamic instability, transfusion reaction, infectious complications, citrate-related nausea and vomiting, respiratory distress and seizure. ${ }^{9}$ Death is rare and is usually due to the underlying disease. However, none of these adverse events occurred in our patient.

\section{CONCLUSION}

This case highlights the importance of awareness of this uncommon but life-threatening adverse event of propranolol-induced circulatory collapse in patients with thyroid crisis associated with pre-existing thyrotoxic cardiomyopathy. Ultra-short acting $\beta$-blockers with high cardio-selectivity should be considered over NCBB in a critical care setting with careful titration and close hemodynamic monitoring. TPE should be considered in thyroid crisis associated with life-threatening symptoms especially in the presence of multi-organ dysfunction or rapid clinical deterioration apart from failure to respond to conventional medical therapy. The duration and frequency of TPE should be individualized. It may be discontinued upon significant clinical improvement and resolution of end organ dysfunction.

\section{Acknowledgments}

The authors thank the Director General of Health Malaysia for his permission to publish this article.

\section{Ethical Consideration}

Patient consent was obtained before submission of the manuscript.

\section{Statement of Authorship}

All authors certified fulfillment of ICMJE authorship criteria.

\section{Author Disclosure}

The authors declared no conflict of interest.

\section{Funding Source}

None.

\section{References}

1. Zainudin S, Hussein Z, Jalaludin MY, et al. A summary of the consensus for the management of thyroid disorders in Malaysia. J ASEAN Fed Endocr Soc. 2012;27(1):40-3. https://doi.org/10.15605/jafes.027.01.06.

2. Pokhrel B, Bhusal K. Thyroid crisis. Treasure Island. StatPearls [Internet]. Treasure Island (FL): StatPearls Publishing; 2019. PMID: 28846289.

3. Abubakar H, Singh V, Arora A, Alsunaid S. Propranolol-induced circulatory collapse in a patient with thyroid crisis and underlying thyrocardiac disease: A word of caution. J Investig Med High Impact Case Rep. 2017;5(4): 2324709617747903. PMID: 29318163. PMCID: PMC5753961. https://doi.org/10.1177/2324709617747903.

4. Satoh T, Isozaki O, Wakino S, et al. 2016 Guidelines for the management of thyroid crisis from The Japan Thyroid Association and Japan Endocrine Society (First Edition). Endocr J. 2016;63(12):1025-64. PMID: 27746415. https://doi.org/10.1507/endocrj.EJ16-0336.

5. Mio Y. New ultra-short-acting beta blockers: Landiolol and esmololthe effects on cardiovascular system. Masui. 2006;55(7):841-8. PMID: 16856544 .

6. Muller C, Perrin P, Faller B, Richter S, Chantrel F. Role of plasma exchange in the thyroid storm. Ther Apher Dial. 2011;15(6):522-31. PMID:22107688. https://doi.org/10.1111/j.1744-9987.2011.01003.x.

7. Schwartz J, Padmanabhan A, Aqui N, et al. Guidelines on the use of therapeutic apheresis in clinical practice-evidence based approach from the writing committee of the American Society for apheresis: The seventh special issue. J Clin Apher. 2016; 31(3):149-62. PMID: 27322218. https://doi.org/10.1002/jca.21470.

8. Ross DS, Burch HB, Cooper DS, et al. 2016 American Thyroid Association Guidelines for diagnosis and management of hyperthyroidism and other causes of thyrotoxicosis. Thyroid. 2016;26(10):1343-421. PMID: 27521067. https://doi.org/10.1089/thy.2016.0229.

9. McLeoad BC. Plasma and plasma derivatives in therapeutic plasmapheresis. Transfusion. 2012;52(Suppl 1):38S-44S. PMID: 22578370. https://doi.org/10.1111/j.1537-2995.2012.03623.x.

Authors are required to accomplish, sign and submit scanned copies of the JAFES Author Form consisting of: (1) Authorship Certification, that authors contributed substantially to the work, that the manuscript has been read and approved by all authors, and that the requirements for authorship have been met by each author; (2) the Author Declaration, that the article represents original material that is not being considered for publication or has not been published or accepted for publication elsewhere, that the article does not infringe or violate any copyrights or intellectual property rights, and that no references have been made to predatory/ suspected predatory journals; (3) the Author Contribution Disclosure, which lists the specific contributions of authors; and (4) the Author Publishing Agreement which retains author copyright, grants publishing and distribution rights to JAFES, and allows JAFES to apply and enforce an Attribution-Non-Commercial Creative Commons user license. Authors are also required to accomplish, sign, and submit the signed ICMJE form for Disclosure of Potential Conflicts of Interest. For original articles, authors are required to submit a scanned copy of the Ethics Review Approval of their research as well as registration in trial registries as appropriate. For manuscripts reporting data from studies involving animals, authors are required to submit a scanned copy of the Institutional Animal Care and Use Committee approval. For Case Reports or Series, and Images in Endocrinology, consent forms, are required for the publication of information about patients; otherwise, appropriate ethical clearance has been obtained from the institutional review board. Articles and any other material published in the JAFES represent the work of the author(s) and should not be construed to reflect the opinions of the Editors or the Publisher. 